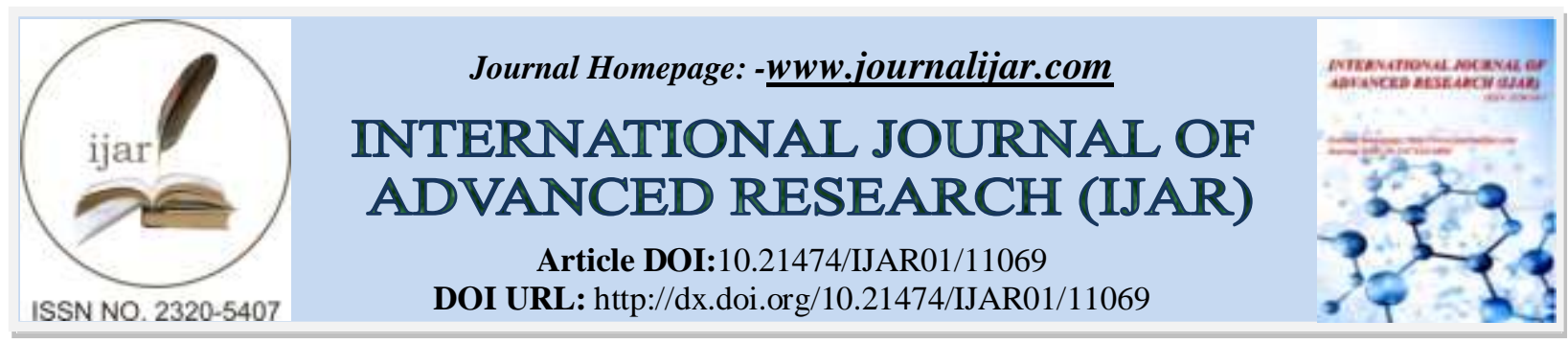

RESEARCH ARTICLE

\title{
ASYMPTOMATICGIANT RIGHT ATRIAL THROMBUS TREATED SUCCESSFULLY WITH
} ANTICOAGULATION

Dinia Mohamed, Khelifi Lamiae and Hatem Saeed

Cardiovascular Department, IBN SINA University Hospital, Rabat, Morocco.

\section{Manuscript Info}

Manuscript History

Received: 01 April 2020

Final Accepted: 05 May 2020

Published: June 2020

Key words:-

Right Atrial Thrombus, Pulmonary

Embolism, Anticoagulation

\begin{abstract}
Right heart thrombi are rare, and are often associated with poor prognosis. They are usually discovered in the setting of acute pulmonary embolism but may be found by chance during a routine echocardiography. Clinical presentations of such thrombi are variable depending on the size of the mass, obstruction of cardiac chambers and embolization to the pulmonary bed. The optimal therapeutic strategy is still a matter of debate and options include anticoagulation, fibrinolysis and surgery.We report the case of a completely asymptomatic 71-yearold patient with history of pacemaker implantation presenting for his yearly check-up. ECG found atrial fibrillation and echocardiography revealed a giant mobile right atrial thrombus $(50 \times 53 \mathrm{~mm})$. Anticoagulation alone resulted in complete disappearance of the thrombus after 1 month without clinical worsening. Despite the successful outcome in this patient, the best therapy for right heart thrombi remains controversial and should be individualized in each patient.
\end{abstract}

Copy Right, IJAR, 2020,. All rights reserved.

\section{Introduction:-}

Right heart thrombi are rare, and are often associated with poor prognosis [1].Their presence represents a serious situation with a major risk of pulmonary embolization and circulatory collapse. Optimal therapeutic strategy is still controversial in the absence of clear guidelines.

\section{Case Report:-}

A 71-year-old patient presented to the cardiology department for his yearly check-up. Three years earlier, he was implanted with a dual-chamber pacemaker for a symptomatic paroxysmal Third-degree atrioventricular block. There was no family history suggestive of a thrombotic disorder. The patient was completely asymptomatic since the pacemaker implantation and did not report any cardio-respiratory symptoms. Physical examination found a fast and irregular heart rate, blood pressure of $110 / 70 \mathrm{mmHg}$ and normal oxygen saturation. ECG showed atrial fibrillation rhythm with rapid ventricular rate. Interrogation of the pacemaker found no modification of stimulation parameters.

Transthoracic echocardiography revealedthe presence of a giant mobile mass suggestive of thrombus occupying a large area of a dilated right atrium. The right ventricle was of normal dimensions with normal systolic function and pulmonary artery pressure was in the normal range.Trans-esophageal echocardiography allowed a better assessment of the mass, measuring 50x53mm with characteristics of a thrombus (figure 1).Computed tomography scan excluded pulmonary embolism and lower limb ultrasound did not find any signs of deep venous thrombosis. Laboratory tests were unremarkable. 
We concluded that atrial fibrillation and the presence of pacemaker leads both contributed to the development of a large right atrial thrombus. Once the diagnosis was made, the question about the optimal therapeutic strategy was raised. Given the hemodynamic stability and the absence of pulmonary embolism, we opted for anticoagulation with a vitamin $\mathrm{K}$ antagonist bridged with heparin as a first line treatment. The patient was closely followed-up, taking into consideration the risk of embolization and the possible need for urgent invasive treatment. Transthoracic echocardiography was done every week and showed progressive decrease in the size of the thrombus with complete disappearance after one month. In this case, anticoagulation alone was successful treating the right atrial thrombus without clinical worsening.

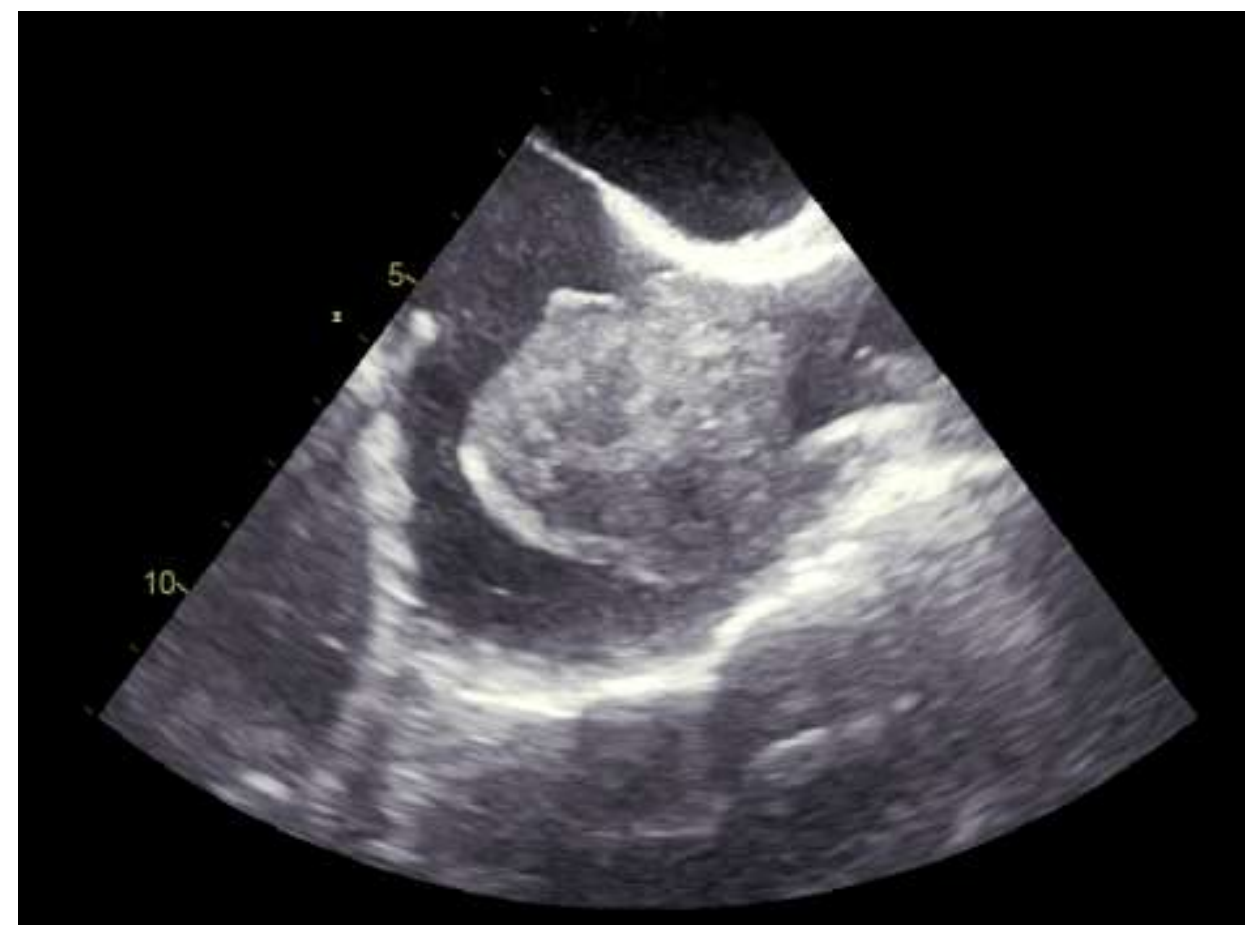

Figure 1:- Trans-esophageal echocardiography showing a large right atrial thrombus (50x53mm).

\section{Discussion:-}

Right heart thrombi (RHT) are rare, and are often associated with poor prognosis [1]. Their prevalence is estimated to be $<4 \%$ of patients with acute pulmonary embolism (PE) and may reach $18 \%$ among patients in the intensive care setting [2].They have been classified according to their morphology, as type A (worm-shaped thrombi) commonly associated with peripheral venous thrombosis and type B (non-specific clots resembling left heart thrombi) usually developed within the right cardiac chambers [3].Numerous conditions are presumed to be predisposing factors for intra-cardiac thrombus development: atrial fibrillation, right atrial enlargement, foreign bodies in the right heart (pacemaker wire, central venous line etc.), atriotomy, right ventricular damage (infarction, congestive cardiomyopathy, chronic right heart failure, Loffler's endocarditis, hypoplastic right heart) [3].

The clinical presentation of RHT is utterly variable, including sudden death, symptomatic non-fatal pulmonary embolism and no symptoms at all. In patients with PE, clinical manifestation differed depending on the presence of such thrombi: dyspnea was more frequent in patients with free-floating thrombi (FFT), whereas chest pain was more frequent in their absence [4].Moreover,in this group,FFT increased the prevalence of cardiogenic shock,cardiac arrest and tachycardia.Transthoracic and Trans-esophagealechocardiography represent the methods of choice for the detection of RHT[1]. Computed tomography is also a useful imaging technique for screening of RHT with excellent sensitivity and negative predictive value [4].Lower limb compression ultrasonography may be helpful to look for deep venous thrombosis being the origin of the RHT.

The presence of right-sided cardiac mobile thrombi may compromise the prognosis. In the ICOPER Registry, the mortality rate was significantly higher in patients presenting with FFT (21\% versus $11 \%$, p=0.03) [1]. It was suggested that the morphology of a right heart thrombus was an important predictor of prognosis. Patients with type 
A thrombi had a very poor short-term prognosis illustrated by a higher incidence and severity of pulmonary embolism and by a worst early mortality rate in comparison to patients with type B thrombi [3]. Although In patients with RHT and pulmonary embolism, the outcome was predominantly related to the hemodynamic and clinical consequences of acute PE itself, and not to the morphology of the thrombus [5].

Patients with RHT represent a therapeutic emergency even if hemodynamically stable because of the major risk of pulmonary embolization and circulatory collapse. Optimal therapy is still a subject of debate and therapeutic options include anticoagulation, fibrinolysis and surgical or percutaneous embolectomy. Kinney and Wright [6]showed a marginally increased survival with anticoagulation when compared to surgical embolectomy or thrombolysis (70\% versus $62 \%$ ). However, in this group, not all patients had PE and the representation of each therapy was unclear. ATHAPAN et al.[7] included 328 patients with RHT, no contraindications to either therapy and an even representation of all three treatments. Anticoagulation alone increased mortality when compared to thrombolysis or surgical embolectomy. Treatment strategy and hemodynamic status of the patient were the only major predictors of survival in this group.

The European Working Group on Echocardiography found interesting results in favor of surgery [3].They reported a lower observed mortality with surgical embolectomy when compared to anticoagulation (27\% versus $>60 \%)$. Their report did not assess thrombolysis as only few patients received it. Surgical embolectomy is supposedly the most definitive therapy for RHT as it promptly clears the clot from the right cardiac chambers and fixes pulmonary artery obstruction. Nonetheless, it is also the most invasive therapy with a necessity of expert surgeons in this procedure. Regarding thrombolysis, Rose et al. [8] performed a pooled analysis of 177 patients with RHT. They showed that thrombolytic therapy was superior to surgical embolectomy (OR for mortality: 2.83 , 95\% CI 1.04-7.69) and to anticoagulation (OR for mortality: 3.03, 95\% CI 1.02-3.125). The greatest worry when using thrombolytic agents is the risk of dislodgement of the clot into the pulmonary artery resulting in massive PE and the risk of major bleeding. In our case, the patient had no contraindication to either therapy and the choice of anticoagulation alone was based on the clinical stability and the absence of pulmonary embolism. However, the patient was strictly followed-up and we were prepared to move on to more aggressive therapy if needed.

\section{Conclusion:-}

In the presence of predisposing factors, right heart thrombi should be looked forsystematically. Their presence has important prognostic and therapeutic implications. The question of the optimal treatment remains to be answered. Therapy should be individualized weighing the risks and benefits of each treatment strategy and taking into account hemodynamic status, bleeding risk and thrombus characteristics.

\section{Conflict of Interest:}

None

\section{References:-}

1. Torbicki A, Galie N, Covezzoli A et al. Right heart thrombi in pulmonary embolism: results from the International Cooperative Pulmonary Embolism Registry. J Am CollCardiol. 2003;41:2245-2251

2. Konstantinides S, Meyer G, Becattini C, et al. 2019 ESC Guidelines for the Diagnosis and Management of Acute Pulmonary Embolism Developed in Collaboration With the European Respiratory Society (ERS). Eur Heart J. 2020; 41(4): 543-603.

3. European Working Group on Echocardiography. The European Cooperative Study on the clinical significance of right heart thrombi. EurHeart J 1989; 10: 1046-1059.

4. Mansencal N, Attias D, Caille V, et al. Computed tomography for the detection of free-floating thrombi in the right heart in acute pulmonary embolism. EurRadiol 2011;21: 240-245.

5. Koć M, Kostrubiec M, Elikowski W, et al. Outcome of patients with right heart thrombi: the Right Heart Thrombi European Registry. EurRespir J 2016;47(3):869-75.

6. Kinney EL, Wright RJ. Efficacy of treatment of patients with echocardiographically detected right-sided heart thrombi: a meta-analysis. Am Heart J 1989; 118: 569-573.

7. Athappan G, Sengodan P, Chacko P, Gandhi S. Comparative efficacy of different modalities for treatment of right heart thrombi in transit: A pooled analysis. Vascular Medicine 2015;20(2): 131-138.

8. Rose PS, Punjabi NM, Pearse DB. Treatment of right heartthromboemboli. Chest 2002; 121: 806-814. 